\title{
Surveillance of infection burden in residential aged care facilities
}

Ching Jou Lim

BPharm(Hons)

PhD Student

Susan C McLellan RN, GradCert InfectControl, GradDipNSInfectControl Infection Prevention Nurse Consultant

Allen C Cheng RACP, MPH, PhD Deputy Head, ${ }^{2}$ and Associate Professor of Infectious Disease Epidemiology ${ }^{3}$

Joanne M Culton RN, GradCert InfectControl Infection Prevention Nurse Consultant

Sneha N Parikh Research Student

Anton Y Peleg* MB BS, PhD, FRACP, Infectious Diseases Physician, ${ }^{4}$ and Senior Lecturer

David C M Kong* BPharm, MPharm, PhD Lecturer

ICentre for Medicine Use and Safety, Monash University,

Melbourne, VIC

2 Infection Prevention and Healthcare Epidemiolog

Unit, Alfred Health

Melbourne, VIC

3 Department of Epidemiology and

Preventive Medicine,

Monash University Melbourne, VIC

4 Infectious Diseases Unit. Alfred Hospital, Melbourne, VIC

5 Microbiology Department Monash University, Melbourne, VIC

* Joint senior authors

david.kong@ monash.edu

anton.peleg@ monash.edu

MJA 2012; 196: 327-331 doi: 10.5694/mjal2.10085 nfection surveillance is a crucial component of an integrated infection control program, providing timely feedback about infection prevention issues that is geared towards decreasing the burden of infection within health care facilities. ${ }^{1}$ Data from overseas suggest there is a considerable infection burden among aged care residents. ${ }^{2-6}$ The Aged Care Standards and Accreditation Agency Ltd has highlighted that surveillance is required in Australian residential aged care facilities (RACFs), but there are few published studies describing the epidemiology of health care-associated infection (HCAI) and antimicrobial use. $^{7,8}$

A recent Australian study highlighted a concern about emergence of antimicrobial-resistant organisms among RACF residents. ${ }^{8}$ The study, however, did not describe the infection burden and the associated antimicrobial prescribing patterns among this population. Although some overseas studies report extensive prescribing of broad-spectrum antibiotics, ${ }^{9-11}$ differences in clinical practice are likely in Australia.

Accordingly, we explored the patterns of common infections and use of antimicrobial agents in four Australian RACFs using internationally recognised criteria. ${ }^{12}$ Recognition of the infection burden among residents in this long-term care setting will help to identify infection prevention priorities and guide antimicrobial stewardship efforts.

\section{Methods}

\section{Study setting}

This study involved four RACFs (150 residential care beds in total) administered by Caulfield Hospital's Residential Care Services in Melbourne, Australia. These nursing

Abstract

Objectives: To explore the burden of illness associated with infectious syndromes and to measure the associated use of antimicrobials in residential aged care facilities (RACFs).

Design, setting and subjects: Retrospective analysis of data for January 2006 to December 2010 from an infection surveillance system covering residents of four co-located RACFs, with a total of 150 residential care beds, in Melbourne, Victoria.

Main outcome measures: Number of episodes and incidence of health careassociated infection ( $\mathrm{HCAl}$ ); rate of antimicrobial use; prescribing concordance with McGeer criteria for infection; frequency of clinical specimen collection.

Results: There were 1114 episodes of an infectious syndrome over 267684 occupied bed-days (OBD), affording an average HCAl rate of 4.16 episodes/ 1000 OBD annually over 5 years ( $95 \% \mathrm{Cl}, 3.92-4.41)$. The mean rate of antimicrobial use was 7.07 courses/1000 OBD (range, 6.71-7.84). Around 40\% of antimicrobial prescribing was for episodes that did not fulfil the McGeer criteria for clinical infection; this included about half of suspected urinary tract and upper respiratory tract infections (URTI), and about one-third of suspected lower respiratory tract and skin infections. Antimicrobials were routinely prescribed for URTI and bronchitis. Of all episodes treated with antimicrobials, $36 \%$ had documentation that a clinical specimen was obtained.

Conclusions: The HCAl rate remained relatively stable over time. Routine surveillance and feedback of infection rates to the facilities did not result in a noticeable decrease of infection burden over time. It is of immediate concern that antimicrobials were being prescribed for a large proportion of suspected infections that did not meet criteria for clinical infection. Opportunities exist to further improve the use of antimicrobials in the RACF setting.

homes provide 24-hour high-level nursing care to elderly residents with multiple disabilities including dementia and psychological disorders. Most $(80 \%)$ residential care beds are for permanent residents, but one facility accommodates transitional care residents (30 care beds; maximum length of stay is generally 3 months). Although the four facilities are co-located on the Caulfield Hospital campus of Alfred Health, medical care is provided by general practitioners. The choices of pharmacy and pathology providers are determined by each GP. Nursing services for these RACFs include a dedicated infection prevention nurse consultant. The study was approved by the human research ethics committees of Alfred Health and Monash University.

\section{Infection surveillance system}

A systematic infection surveillance system was introduced across the RACFs; initially (from 2003), to monitor the incidence of urinary tract infection (UTI) and conjunctivitis, then later (from mid 2005), other HCAIs were included. HCAI refers to infection occurring at least 48 hours after admission to an RACF with new or acutely worsening symptoms that fulfil criteria for site-specific infection. These definitions, known as the McGeer criteria, ${ }^{12}$ refer to a set of clinical and other criteria intended specifically for use in longterm care facilities (see online-only Appendix at mja.com.au). They have been widely accepted as an epidemiological tool for interfacility comparison of infection rates, both locally and globally. $3,5,7,13,14$ 
To maintain consistency, infection surveillance activity is coordinated by an experienced infection prevention nurse consultant (IPNC). As routine practice, senior nursing staff from the respective RACFs notified the IPNC, using a standard data collection form, when a resident had possible signs or symptoms of infection. Information such as the resident's demographic data, signs and symptoms observed and other relevant infection management data (for instance, types of clinical specimens, antimicrobial prescribed and microbiological results) was recorded. The IPNC then reviewed the resident's medical records to assess whether they met the McGeer criteria. Episodes that were validated based on the McGeer criteria were included in the infection surveillance database, and a monthly feedback on infection rates was provided to the individual RACFs. Data on antimicrobial use were retained for suspected infections that did not fulfil the McGeer criteria, and these were appropriately archived from 2009 onwards.

\section{Data collection}

We analysed the infection surveillance database from 2006 to 2010 to determine the overall prevalence and trends over time of HCAIs. Data collection was based on the validated episodes of HCAI being recorded in the surveillance database over the 5 years. Data on antimicrobial use were only available from 2009 onwards. Accordingly, all episodes of suspected infection (those fulfilling and not fulfilling McGeer criteria) between 2009 and 2010 were reviewed to determine patterns of antimicrobial use.

\section{Data analysis}

We used descriptive statistics to summarise the data - number and percentage for categorical variables, and mean for continuous variables. Rate of infection was calculated as the number of episodes of infections per 1000 occupied bed-days (OBD), whereas antimicrobial prescribing was described in courses per 1000 OBD. We calculated the 95\% confidence interval (CI) for the infection rate using OpenEpi version 2.3.1 (Open Source Epidemiologic Statistics for Public Health, version 2.3.1; http:// www. openepi.com), assuming a Pois-
1 Proportion and incidence rate of major types of health care-associated infection in four Melbourne RACFs, January 2006 to December 2010

\begin{tabular}{|c|c|c|}
\hline Type of infection & $\begin{array}{c}\text { Overall infections, } \\
\text { no. (\%) }\end{array}$ & $\begin{array}{l}\text { Incidence (episodes/ } \\
1000 \text { OBD [95\% CI]) }\end{array}$ \\
\hline Urinary tract infection (UTI*) & $410(36.8 \%)$ & $1.53(1.39-1.69)$ \\
\hline $\begin{array}{l}\text { Upper and lower respiratory tract infection } \\
\left(\text { URTI }^{\dagger} \text { and } L R T I^{\ddagger}\right)\end{array}$ & 300 (26.9\%) & $1.12(1.00-1.25)$ \\
\hline Eye, ear, mouth infection & $190(17.1 \%)$ & $0.71(0.61-0.82)$ \\
\hline Skin and soft tissue infection (SSTI $)$ & $164(14.7 \%)$ & $0.61(0.52-0.71)$ \\
\hline Gastroenteritis & $41(3.7 \%)$ & $0.15(0.11-0.21)$ \\
\hline Systemic infection" & $9(0.8 \%)$ & $0.03(0.02-0.06)$ \\
\hline Overall & 1114 (100\%) & $4.16(3.92-4.41)$ \\
\hline
\end{tabular}

RACF $=$ residential aged care facility. OBD = occupied bed-days. * Both indwelling-catheter (IDC)-related UTI and non-IDC-related UTI. † Common cold or pharyngitis and influenza-like illness. $\ddagger$ Bronchitis or tracheobronchitis and pneumonia. $\oint$ Cellulitis or wound infection, fungal infection, herpes zoster infection and scabies. १९ Unexplained febrile illness and bloodstream infection. son distribution. We used the monthly recorded rates of infection that conformed to the McGeer criteria over the 5-year period to construct a statistical control chart, using IBM SPSS Statistics 19 (IBM Corporation, New York, NY, USA). A proportion ( $p$ ) control chart was selected to account for the proportion of infections in the studied RACF population over time. ${ }^{15}$ Infection rates above the upper control limit may indicate a potential outbreak.

\section{Results}

\section{Common infectious syndromes}

Over 5 years, from January 2006 to December 2010, 1114 infections that fulfilled the McGeer criteria were recorded over 267684 OBD (98\% bed occupancy) in the four RACFs studied. The estimated mean HCAI rate was 4.16 episodes per 1000 OBD annually (95\% CI, 3.92-4.41). The infection rates across the four RACFs were similar, with yearly incidence ranging from 3 to 5.5 episodes per 1000 OBD. The distribution of validated HCAIs is shown in Box 1.

The four most common infectious syndromes, representing $89 \%$ of HCAIs, were UTI, lower respiratory tract infection (LRTI), skin and soft tissue infection (SSTI) and eye infection $(1.53,0.97,0.61$, and 0.57 per 1000 OBD, respectively). Pneumonia $(0.07 / 1000$ OBD) and gastroenteritis $(0.15 / 1000$ OBD) constituted less than $6 \%$, and only one bloodstream infection was documented.

\section{Proportion control chart showing proportion of infections (episodes/OBD) per} month in four Melbourne RACFs, January 2006 to December $2010(n=1114)$

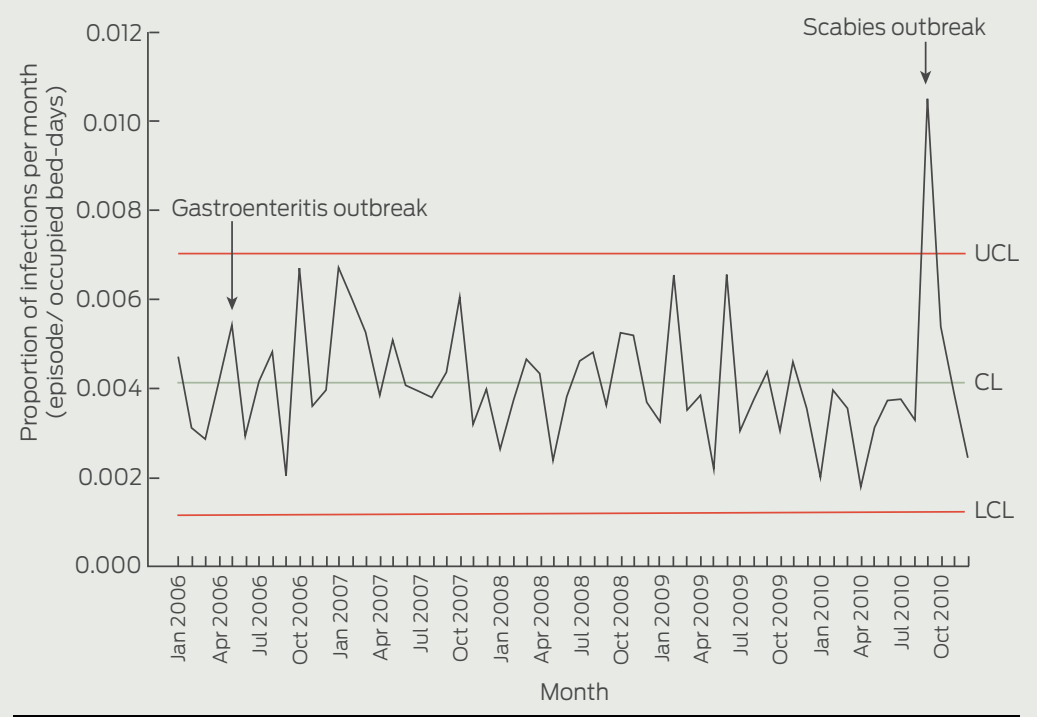

$\mathrm{OBD}=$ occupied bed-days. $\mathrm{RACF}=$ residential aged care facility. $\mathrm{CL}=$ centre line. $\mathrm{UCL}=$ upper control limit (mean + 3SD). LCL= lower control limit (mean - 3SD). 
The monthly trend of infections within the population was assessed using a $p$ control chart (Box 2); the monthly proportion significantly exceeded the upper control limit in September 2010, owing to a scabies outbreak. While the statistical control chart suggested some fall in infection rates at the end of the study (eg, with eight points below the mean, January 2010 to August 2010), the overall trend in the rate of infections showed a small non-significant decrease over the 5-year period.

\section{Antimicrobial use and prescribing patterns}

In 2009-2010, 662 episodes of suspected infection were notified, and patients were prescribed at least one antimicrobial agent in 659 episodes. Of these, 247 episodes (37\%) did not fulfil the McGeer criteria for clinical infection. The McGeer criteria were not met in 141/288 reported episodes of UTI (49\%), 48/147 episodes of LRTI (33\%), 24/47 episodes of URTI and 27/

\begin{tabular}{|c|c|}
\hline $\begin{array}{l}\text { Type of } \\
\text { antimicrobial }\end{array}$ & $\begin{array}{c}\text { Courses prescribed, } \\
\text { no. }(\%)\end{array}$ \\
\hline Cephalexin & $187(24.8 \%)$ \\
\hline Trimethoprim & $103(13.6 \%)$ \\
\hline $\begin{array}{l}\text { Amoxycillin- } \\
\text { clavulanic acid }\end{array}$ & $97(12.8 \%)$ \\
\hline Roxithromycin & $83(11.0 \%)$ \\
\hline Chloramphenicol & $57(7.5 \%)$ \\
\hline Amoxycillin & $56(7.4 \%)$ \\
\hline Ceftriaxone & $21(2.8 \%)$ \\
\hline Norfloxacin & $16(2.1 \%)$ \\
\hline Erythromycin & $15(2.0 \%)$ \\
\hline Others & 120 (15.9\%) \\
\hline Overall & $755(100 \%)$ \\
\hline
\end{tabular}

83 episodes of skin infection. For the 141 episodes of UTI that did not fulfil the McGeer criteria, the most frequently reported clinical features were altered mental or functional status and change in urine character. In $20 \%$ (28) of these episodes, patients were prescribed antimicrobials for asymptomatic bacteriuria. With respect to the 72 respiratory tract episodes that did not meet the McGeer criteria and for which patients received antimicrobials, the most common symptom was cough, documented for $56 \%$ (27/ 48) of bronchitis episodes and 59\% $(13 / 22)$ of influenza-like illnesses. Fever was documented in only nine of the 34 episodes of influenza-like illness. Fever was a clinical feature in only $13 \%$ of all suspected infections $(84 / 662 ; 17 \%$ of validated episodes and $6 \%$ of unvalidated episodes).

Between 2009 and 2010, the mean rate of antimicrobial use was 7.07 courses per 1000 OBD (range, 6.717.84 courses per 1000 OBD for the four RACFs). A total of 755 courses of antimicrobials were prescribed, of which $86.2 \%$ were administered orally, $9.4 \%$ were administered topically and $4.4 \%$ were given parenterally. Almost all were antibacterials; $1.5 \%$ were antiviral or antifungal agents.

Cephalexin, trimethoprim, amoxycillin-clavulanic acid and roxithromycin accounted for $62 \%$ of all the courses prescribed (Box 3). UTI was the most common indicator for antimicrobial use (in $41 \%$ of courses), followed by LRTI (in 26\%), SSTI (in $12 \%$ ), URTI (in $8 \%$ ) and eye infection (in 8\%). Box 4 shows antimicrobial prescribing patterns for three major sites of infection (ie, UTI, LRTI and URTI combined, and SSTI). Only about $5 \%$ of antimicrobials were prescribed for other indications such as ear and mouth infection, gastroenteritis, bloodstream infection and unexplained febrile illness. Around 11\% of all antimicrobials prescribed for UTIs were broad-spectrum antibiotics, which included norfloxacin, ciprofloxacin, ceftriaxone and gentamicin. Indwelling catheter-related (IDC) UTI contributed to about $20 \%$ of all episodes of UTI; prescribing patterns for both non-IDC and IDC UTIs were similar. Antimicrobials were routinely prescribed for URTI and bronchitis (constituting up to $31 \%$ of overall antimicrobial use). Similar prescribing patterns were observed for episodes that fulfilled McGeer criteria and those that did not.

Of 659 episodes for which patients were treated with antimicrobials, only 238 episodes (36\%) had documentation that clinical specimens were obtained. Clinical specimens were infrequently collected for most types of infection (Box 5). Conversely, collection of urine specimens was documented for $72.2 \%$ of UTI episodes.

\section{Discussion}

The incidence of HCAIs from 2006 to 2010 in four RACFs in Melbourne was comparable to rates reported in the literature, however, despite feedback of infection rates to staff, we did not find evidence of notable improvement in infection burden over time. We found a significant burden of antimicrobial use and identified URTI, bronchitis and asymptomatic bacteriuria as areas where use was likely to be inappropriate.

\section{Commonly prescribed antimicrobials, by site of infection}

\section{A Urinary tract infection}

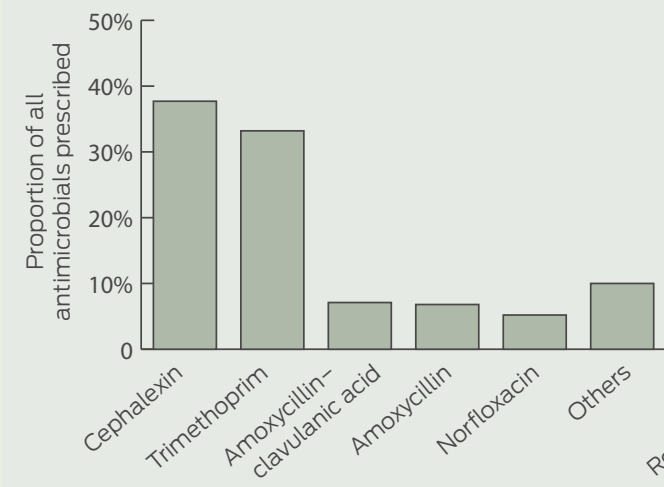

B Respiratory tract infection

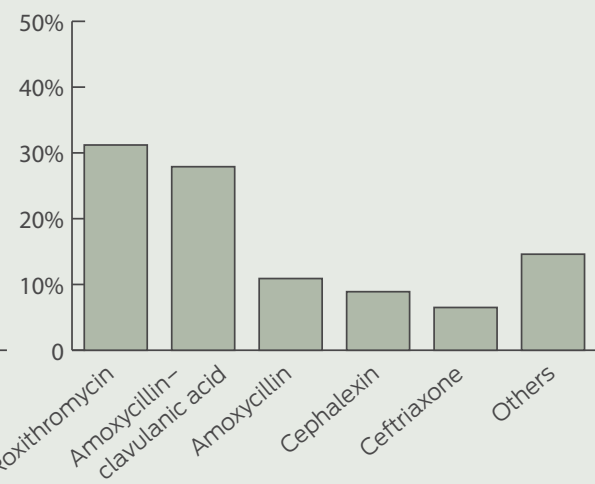

C Skin and soft tissue infection

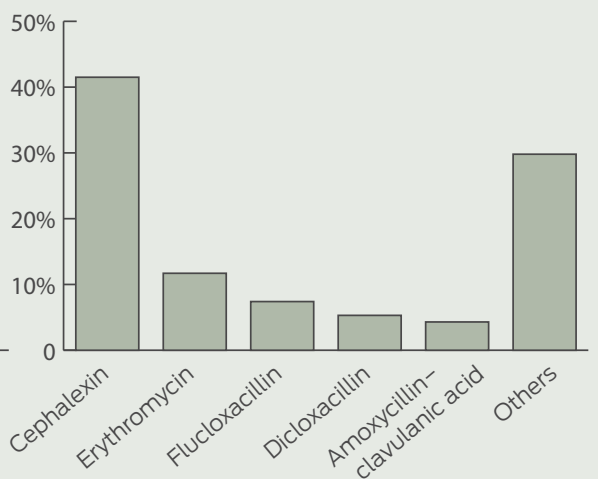




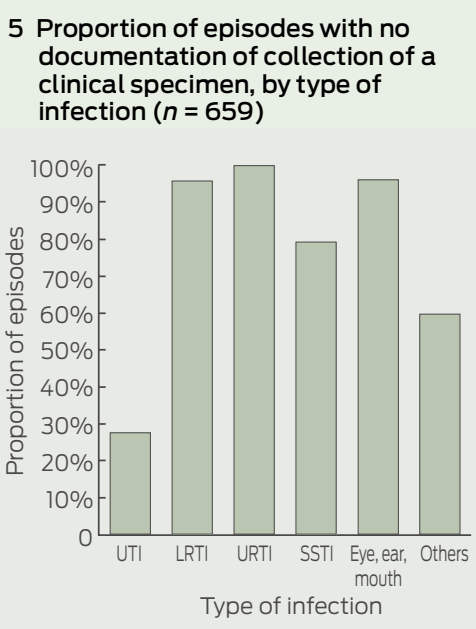

$\mathrm{UTI}=$ urinary tract infection. $\mathrm{LRTI}=$ lower respiratory tract infection. URTI = upper respiratory tract infection. SSTI $=$ skin and soft tissue infection.

Our results indicate that systematic infection surveillance can be established and sustained in Australian RACFs using internationally established criteria, ${ }^{12}$ although there are currently no systematic surveillance systems for infection in RACFs in any Australian jurisdiction. The only two published Australian studies that have examined HCAI rates in this setting were conducted over a 9 -year period in a Sydney network and as a 6-month pilot study in a rural Victorian region. ${ }^{7,16}$ Both studies used the McGeer criteria and reported rates of infection at 3.1 and 2.2 infections per 1000 OBD, respectively. These rates are of the same magnitude as infection rates reported in studies from the United States and European countries of 1.5-9.5 infections per 1000 OBD, based on the McGeer criteria. ${ }^{2-6,14}$ The average rate and types of HCAI in our study were generally comparable to the reported literature, except for the rate of pneumonia, which was considerably lower than that in some studies. ${ }^{11,17,18}$ We found that chest $x$-rays, required to fulfill criteria for pneumonia, were often not performed in this patient population, suggesting that the low rate may reflect differences in clinical practice, rather than a true difference in pneumonia rate. The rate of gastroenteritis (0.15 episodes per 1000 OBD) was lower than that reported in one other local study (0.29-1.42 episodes per 1000 OBD). ${ }^{19}$
Importantly, the rates of HCAI remained relatively stable over time, with the exception of two outbreaks reported in 5 years, suggesting that routine surveillance and feedback of infection-related issues did not result in noticeable improvement in preventing infections. Routine surveillance without more proactive measures may not effectively improve the burden of HCAIs and antimicrobial use over time. Most infection prevention guidelines are designed for use in hospitalised patients with a high prevalence of invasive devices. Work is required in developing and validating infection prevention policies for the RACF setting.

There was a significant burden of oral antimicrobial use in this population, with a high proportion of treated episodes not meeting infection criteria, reflecting the difficulties in clinically assessing this patient population. The antimicrobial burden in the RACFs we studied (7.07 courses per 1000 OBD) was mid-range compared with those reported in overseas studies (2-15 courses per 1000 OBD). ${ }^{10,17,20,21}$ Data from Australian RACFs for benchmarking are lacking. The rate of antimicrobial use cannot easily be compared with hospitalbased and community-based studies, owing to differences in surveillance methods. Nonetheless, if the average duration of an antimicrobial course was around 7 days, the use rate could be converted and would approximate 50 defined daily doses (DDD) per 1000 OBD, which is higher than the estimated use in the community (about 25 DDD/1000 population days $)^{22}$ but much lower than the estimated use in large Australian hospitals (about 1000 DDD/1000 OBD). ${ }^{23}$

In our study, the considerable proportion of antimicrobials prescribed for episodes that did not meet the McGeer criteria was similar to that reported elsewhere. ${ }^{8,20}$ Although the McGeer criteria are well established for epidemiological comparison, antimicrobial use in patients not meeting these criteria may not always be inappropriate. Nevertheless, it was noted that all episodes of URTI and nearly all of bronchitis led to routine prescribing of antimicrobials without further diagnostic investigation to confirm bacterial aetiology, contrary to recommendations in the national
Antibiotic Therapeutic Guidelines. ${ }^{24}$ Similarly, the use of antimicrobials for asymptomatic bacteriuria is not recommended. The prescribing of antimicrobials for asymptomatic bacteriuria is of particular concern because the emergence of multiresistant organisms in the RACF setting ${ }^{25,26}$ is often attributable to extensive or inappropriate use of antimicrobials and may result in the RACFs becoming a reservoir for multiresistant organisms. Barriers to improving the quality of antimicrobial prescribing include the complexities of assessing elderly patients due to atypical illness presentation, lack of timely investigations to guide therapy, and the large number of health care providers involved in the care of RACF residents.

Several sources suggest that fluoroquinolones were the most commonly prescribed antibiotics for common infections in the RACF setting overseas. ${ }^{9,10,27,28}$ However, our data show that cephalexin and trimethoprim were used to a much higher extent, and the use of fluoroquinolones or other broad-spectrum antibiotics was low. The low use of fluoroquinolones is likely to reflect, at least in part, restricted indications under the Australian Pharmaceutical Benefits Scheme. The high use of chloramphenicol, on the other hand, was due to the widespread prescribing of topical chloramphenicol eye preparations for conjunctivitis. Pleasingly, in most cases, the empirical prescribing pattern for UTIs was in line with the national guidelines. ${ }^{24}$

Routine ordering of microbiological tests is not always practical in the RACF setting. This reflects difficulties in obtaining specimens, the involvement of multiple laboratories servicing these institutions and the lack of timely results that inform prescribing. In addition to difficulties in clinical diagnosis, the lack of clinical cultures also hinders the monitoring of antimicrobial susceptibility trends among this population. Accordingly, alternative approaches such as periodic surveillance of antimicrobial resistance patterns may be more feasible in this setting.

There were several limitations to our study. The results from four RACFs located on a hospital campus may not be generalisable to other 
RACF populations. However, we noted that most subjects were residents of the facilities, with little traffic of patients from the hospital. The rates of infection remained relatively constant and were comparable to those reported in another longitudinal study in Australia. ${ }^{7}$ Although the McGeer criteria are the only internationally recognised epidemiological definitions of infection, they may not be completely specific or sensitive for microbiologically confirmed infection. We were not able to analyse the epidemiology of bacterial pathogens, owing to the small number of cultures performed. Recent point prevalence studies have assisted in defining the prevalence of colonisation with antimicrobial-resistant pathogens, ${ }^{8}$ but data are also required on their contribution to the burden of infection to inform antibiotic prescribing policies.

Work is required in developing policies to prevent infection among RACF residents, to explore the epidemiology of antimicrobial resistance in clinical infections, and in developing antimicrobial stewardship initiatives appropriate to this primary care setting.

Acknowledgements: We thank Denis Spelman, Jacqueline Kennon, Kerrie Watson, the Alfred Infection Prevention and Hospital Epidemiology Unit, and Victorian Infection Control Surveillance Project. We also thank Andrew Perta, Gabrielle Melican and the nursing home managers (Kylie-Ann Thomas, Olga Rozjimalina, Galina Pudel and Wima Edwards) for their input.

Competing interests: No relevant disclosures.

Received 12 Jan 2012, accepted 22 Feb 2012.

1 Hughes JM. Study on efficacy of nosocomial infection control (SENIC Project): results and implications for the future. Chemotherapy 1988; 34: 553-561.
2 Darnowski SB, Gordon M, Simor AE. Two years of infection surveillance in a geriatric long-term care facility. Am J Infect Control 1991; 19: 185-190.

3 Engelhart ST, Hanses-Derendorf L, Exner M, Kramer MH. Prospective surveillance for healthcare-associated infections in German nursing home residents. J Hosp Infect 2005; 60: 46-50.

4 Eriksen HM, Koch AM, Elstrøm P, et al. Healthcare-associated infection among residents of long-term care facilities: a cohort and nested case-control study.J Hosp Infect 2007: 65: 334-340.

5 Roberts C, Roberts J, Roberts R. Survey of healthcare-associated infection rates in a nursing home resident population. J Infect Prev 2010; 11 : 82-86.

6 Schulz M, Mielke M, Wischnewski N. Clusters of infectious diseases in German nursing homes: observations from a prospective infection surveillance study, October 2008 to August 2009. Euro Surveill 2011; 16. pii: 19881.

7 Forrest J, Tucker A, Brnabic AJM. A 9-year infection-control surveillance program in Sydneybased residential aged-care facilities. Healthcare Infection 2011; 16: 108-114.

8 Stuart RL, Kotsanas D, Webb B, et al. Prevalence of antimicrobial-resistant organisms in residential aged care facilities. Med J Aust 2011; 195: 530-533.

9 Loeb MB, Craven S, McGeer AJ, et al. Risk factors for resistance to antimicrobial agents among nursing home residents. Am J Epidemiol 2003; 157: 40-47.

10 Mylotte JM, Keagle J. Benchmarks for antibiotic use and cost in long-term care. J Am Geriatr Soc 2005; 53: 1117-1122.

11 Pettersson E, Vernby A, Molstad S, Lundborg CS. Infections and antibiotic prescribing in Swedish nursing homes: a cross-sectional study. Scand J Infect Dis 2008; 40: 393-398.

12 McGeer A, Campbell B, Emor TG, et al. Definitions of infection for surveillance in long-term care facilities. Am J Infect Control 1991; 19: 1-7.

13 Moro ML, Mongardi M, Marchi M, Taroni F. Prevalence of long-term care acquired infections in nursing and residential homes in the EmiliaRomagna region. Infection 2007; 35: 250-255.

14 Stevenson KB, Moore J, Colwell H, Sleeper B. Standardized infection surveillance in long-term care: interfacility comparisons from a regional cohort of facilities. Infect Control Hosp Epidemiol 2005; 26: 231-238.

15 Benneyan JC. Statistical quality control methods in infection control and hospital epidemiology, part I: Introduction and basic theory. Infect Control Hosp Epidemiol 1998; 19: 194-214.
16 Smith M, Bull AL, Richards M, et al. Infection rates in residential aged care facilities, Grampians region, Victoria, Australia. Healthcare Infection 2011; 16: 116-120.

17 Loeb M, McGeer A, McArthur M, et al. Risk factors for pneumonia and other lower respiratory tract infections in elderly residents of long-term care facilities. Arch Intern Med 1999; 159: 2058-2064.

18 Muder RR. Pneumonia in residents of long-term care facilities: epidemiology, etiology, management, and prevention. Am J Med 1998; 105: 319-330.

19 Kirk MD, Moffatt CRM, Hall GV, et al. The burden of infectious gastroenteritis in elderly residents and staff of long-term care facilities, Australia. Infect Control Hosp Epidemiol 2010; 31: 860-863.

20 Loeb M, Simor AE, Landry L, et al. Antibiotic use in Ontario facilities that provide chronic care. J Gen Intern Med 2001; 16: 376-383.

21 Mylotte JM. Antimicrobial prescribing in longterm care facilities: prospective evaluation of potential antimicrobial use and cost indicators. Am J Infect Control 1999; 27: 10-19.

22 McManus P, Hammond ML, Whicker SD, et al. Antibiotic use in the Australian community, 19901995. Med J Aust 1997; 167: 124-127.

23 National antimicrobial utilisation surveillance program: annual report 2009-2010. Department of Health, Government of South Australia. http:// www.health.sa.gov.au/INFECTIONCONTROL/ Default.aspx?PageContent|D=65\&tabid=199 (accessed Jan 2012)

24 Antibiotic Guidelines Sub-Committee. Therapeutic guidelines: antibiotic (version 14). Therapeutic Guidelines Limited and Victorian Drug Usage Advisory Committee, 2010. http:// www.tg.org.au (accessed Jan 2012).

25 Lautenbach E, Marsicano R, Tolomeo P, et al. Epidemiology of antimicrobial resistance among gram-negative organisms recovered from patients in a multistate network of long-term care facilities. Infect Control Hosp Epidemiol 2009; 30: 790-793.

26 O'Fallon E, Pop-Vicas A, D’Agata E. The emerging threat of multidrug-resistant gram-negative organisms in long-term care facilities. J Gerontol A Biol Sci Med Sci 2009; 64: 138-141.

27 D'Agata E, Mitchell SL. Patterns of antimicrobial use among nursing home residents with advanced dementia. Arch Intern Med 2008; 168: 357-362.

28 Pakyz AL, Dwyer LL. Prevalence of antimicrobial use among United States nursing home residents: results from a national survey. Infect Control Hosp Epidemiol 2010; 31: 661-662. 
Research

\begin{tabular}{|c|c|c|}
\hline Type & Subtype & Criteria to be met \\
\hline \multirow[t]{2}{*}{ UTI } & No indwelling catheter & $\begin{array}{l}\text { At least three of: } \\
\text { a. Fever } \geqslant 38^{\circ} \mathrm{C} \text { or chills } \\
\text { b. New or increased symptoms on urination (burning pain, frequency or urgency) } \\
\text { c. New flank or suprapubic pain or tenderness } \\
\text { d. Change in character of urine (new bloody urine, foul smell, amount of sediment, new pyuria, or microscopic haematuria) } \\
\text { e. Worsening of mental or functional status (may be new or increased incontinence) }\end{array}$ \\
\hline & $\begin{array}{l}\text { Indwelling catheter } \\
\text { in situ }\end{array}$ & $\begin{array}{l}\text { At least two of: } \\
\text { a. Fever } \geqslant 38^{\circ} \mathrm{C} \text { or chills } \\
\text { b. New flank or suprapubic pain or tenderness } \\
\text { c. Change in character of urine (new bloody urine, foul smell, amount of sediment, new pyuria, or microscopic haematuria) } \\
\text { d. Worsening of mental or functional status }\end{array}$ \\
\hline URTI & Influenza-like illness & $\begin{array}{l}\text { At least two of: } \\
\text { a. Runny nose or sneezing } \\
\text { b. Stuffy nose } \\
\text { c. Sore throat or hoarseness or difficulty in swallowing } \\
\text { d. Dry cough } \\
\text { e. Swollen or tender glands in the neck } \\
\text { Both criteria must be met: } \\
\text { 1. Fever } \geqslant 38^{\circ} \mathrm{C} \\
\text { 2. At least three of the following } \\
\text { a. Chills } \\
\text { b. New headache or eye pain } \\
\text { c. Myalgias } \\
\text { d. Malaise or loss of appetite } \\
\text { e. Sore throat } \\
\text { f. New or increased dry cough }\end{array}$ \\
\hline \multirow[t]{2}{*}{ LRTI } & $\begin{array}{l}\text { Bronchitis or } \\
\text { tracheobronchitis }\end{array}$ & $\begin{array}{l}\text { At least three of: } \\
\text { a. New or increased cough } \\
\text { b. New or increased sputum production } \\
\text { c. Fever } \geqslant 38^{\circ} \mathrm{C} \\
\text { d. Pleuritic chest pain } \\
\text { e. New or increased findings on chest examination (rales, rhonchi, wheezes, bronchial breathing) } \\
\text { f. Respiratory rate }>25 \text { per minute, or new or increased shortness of breath, or worsening mental or functional status } \\
\text { Diagnosis can be made only if no chest film obtained or if a radiograph failed to confirm pneumonia. }\end{array}$ \\
\hline & Pneumonia & $\begin{array}{l}\text { Both criteria must be met: } \\
\text { 1. Chest x-ray demonstrates pneumonia, probable pneumonia, or presence of new infiltrate } \\
\text { 2. At least two signs or symptoms described under "bronchitis or tracheobronchitis" }\end{array}$ \\
\hline
\end{tabular}




\section{Appendix The "McGeer criteria"* _ continued}

Eye, ear nose and

mouth

infection

Ear infection

Mouth or periora

infection

Sinusitis

SSTI Cellulitis or soft tissue or wound infection

Fungal

Herpes zoster or herpes simplex

Scabies

\section{GIT}

infection

Gastroenteritis

Systemic

Bloodstream infection

infection
One criterion must be met:

a. Pus appearing from one or both eyes for at least 24 hours

b. New or increased conjunctival redness (with or without itching or pain) present for at least 24 hours

One criterion must be met:

a. Diagnosed by a physician

b. New drainage from one or both ears (non-purulent drainage must be accompanied by ear pain or redness)

Must be diagnosed by a physician or a dentist

Must be diagnosed by a physician

One criterion must be met:

1. Pus present at wound, skin or soft tissue site

2. At least four of:

a. Fever $\geqslant 38^{\circ} \mathrm{C}$ or worsening mental or functional status; and/or, at affected site -

presence of new or increasing:

b. Heat

c. Redness

d. Swelling

e. Tenderness or pain

f. Serous drainage

Both criteria must be met:

1. A maculopapular rash

2. Either physician diagnosis or laboratory confirmation

Both criteria must be met:

1. A vesicular rash

2. Either physician diagnosis or laboratory confirmation

Both criteria must be met:

1. A maculopapular or itching rash

2. Either physician diagnosis or laboratory confirmation

One criterion must be met:

1. Two or more loose or watery stools above what is normal for the resident within 24 hours

2. Two or more episodes of vomiting in a 24-hour period

3. Both of:

a. A stool culture positive for a pathogen (Salmonella spp., Shigella spp., E. coli 0157: H7, Campylobacter spp.) or a toxin assay positive for $C$. difficile toxin

b. At least one compatible symptom or sign (nausea, vomiting, abdominal pain or tenderness, diarrhoea)

One criterion must be met:

1. Two or more blood cultures positive for the same organism

2. A single blood culture documented with an organism thought not to be a contaminant, and at least one of:

a. Fever $\geqslant 38^{\circ} \mathrm{C}$

b. New hypothermia $<34.5^{\circ} \mathrm{C}$

c. Hypotension (systolic blood pressure $<90 \mathrm{mmHg}$ or a drop of $>30 \mathrm{mmHg}$ from baseline)

d. Worsening mental or functional status

Unexplained febrile illness period, with noknown cause

$\mathrm{UTI}=$ urinary tract infection. $\mathrm{URTI}=$ upper respiratory tract infection. $\mathrm{LRTI}=$ lower respiratory tract infection. SSTI = skin and soft tissue infection. GIT = gastrointestinal tract.

* Information in this table has been reproduced from the American Journal of Infection Control (McGeer A, Campbell B, Emor TG, et al. Definitions of infection for surveillance in

long-term care facilities. Am J Infect Control 1991; 19: 1-7) with permission from Elsevier.[Office use only: NOTE: Permission agreement requires online link to homepage of

Am J Infect Control. Add in web version. 